\title{
基于多感受野拉普拉斯生成对抗网络的单幅图像超分辨率重建算法
}

\author{
周勋，杨德刚，冯䩀 \\ (重庆师范大学计算机与信息科学学院 重庆 400000) \\ (460494731@qq.com)
}

\begin{abstract}
摘 要: 目前深度学习模式下的图像超分辨率重建存在对纹理感知不够精确、重建图像不够真实等问题, 为了改善 重建图像质量，提出一种基于多感受野拉普拉斯生成对抗网络的单幅图像超分辨率算法. 首先，利用多感受野特征 提取、可分离拉普拉斯滤波和复合残差密集块构建生成网络，使网络提取更全面的图像信息; 其次，利用多维软标签 对抗网络, 可使生成对抗网络更容易训练且重建图像纹理更加丰富; 最后, 网络预训练采用 $L_{1}$ 损失函数和 VGG 低层 特征, 使重建图像获取整体特征, 训练使用 VGG 高层特征、Charbonnier 损失和生成损失, 使重建结果更加精细, 纹 理更加充分. 实验使用 Div2k 和 Flickr2K 进行模型训练, 使用 Set5 等数据集进行测试. 结果表明, 该算法比 USRNet 等相关算法的网络规模减小 $40 \%$, 感知指数比 USRNet 平均降低 $0.76 \%$, 图像重建结果具有更多细节且真实性更强.
\end{abstract}

关键词: 生成对抗网络; 图像超分辨率; 深度卷积; 拉普拉斯滤波

中图法分类号: TP391.41

DOI: 10.3724/SP.J.1089.2021.18763

\section{Single Image Super-Resolution Reconstruction Algorithm with Generative Adversarial Network Based on Multi-Size Convolution and Laplacian Filtering}

\author{
Zhou Xun, Yang Degang ${ }^{*}$, and Feng Ji \\ (College of Computer and Information Science, Chongqing Normal University, Chongqing 400000)
}

\begin{abstract}
At present, the image super-resolution reconstruction algorithm using deep learning still faces problems such as insufficient texture perception and insufficient realism of the reconstructed image. In order to improve the quality of the reconstructed image, a single image super-resolution reconstruction algorithm with generative adversarial network based on multi-size convolution and Laplacian filtering is proposed. First, multi-size convolution feature extraction, separable Laplacian filtering and composite residual dense block are used to build a generation network which can extract more comprehensive image information. Secondly, multi-dimensional soft labels are utilized to construct the adversarial network, which can train the generative adversarial network easily and reconstruct image texture richly. Finally, the $L_{1}$ loss function and the VGG low-level features are taken to obtain the overall features in the pre-training stage and the VGG high-level features, Charbonnier loss, and generative loss are used to make the reconstruction result more meticulously during training. Div2k and Flickr2K are chosen for model training, and Set5 and other data sets are used for testing. The experiment results show that the network size of this algorithm is $40 \%$ less than USRNet and other related algorithms, and the perceptual index is $0.76 \%$ lower than USRNet on average. The reconstruction result has more details and is more authentic.
\end{abstract}

收稿日期：2020-11-05; 修回日期：2021-05-30. 基金项目：国家自然科学基金(11471061); 重庆市自然科学基金(cstc2018jcyjAX0470); 教育部人文社会科学研究项目(18XJC880002); 重庆市教委科学技术研究项目(KJQN201800539). 周勋(1997一), 男, 硕士研究生, 主 要研究方向为人工智能、神经网络、深度学习; 杨德刚(1976一), 男, 博士, 教授, 硕士生导师, CCF 会员, 论文通讯作者, 主要研究 方向为人工智能、神经网络、非线性动力学系统; 冯䩀(1986-), 男, 博士, 讲师, CCF 会员, 主要研究方向为机器学习、数据挖掘. 
Key words: generative adversarial network; image super-resolution; depth convolution; Laplacian filtering

图像超分辨率(super resolution, SR)是将一幅 低分辨率(low resolution, LR)图像修复或重建成高 分辨(high resolution, HR)图像的过程. 在计算机视 觉领域, SR 能在不更换设备的基础上获得更好的 影像效果，因而备受关注，并广泛应用于如卫星成 像 ${ }^{[1]}$ 、医学成像 ${ }^{[2]}$ 和人脸识别 ${ }^{[3]}$ 等领域.

早在 20 世纪 60 年代, Harris ${ }^{[4]}$ 就提出了 SR 技 术, 后人基于其思想提出频谱外推法进行 SR 重建 并获得普遍认可. 到了 20 世纪 80 年代, SR 技术得 到了进一步的发展，基于重构的算法成为当时的 主流算法, 它主要分为频域算法和空域算法. 消混 叠重建算法是频域算法中具有代表性的一个, 其 具有理论简单、可并行的特点; 空域算法具有先验 约束能力，最具有代表性的算法为凸集投影法 (projections onto convex sets, POCS) 和最大后验概 率(maximum a posterior, MAP). 到了 21 世纪初, SR 技术转换思路, 创新地提出基于样例学习的算 法, 如 Example-based SR 算法 ${ }^{[5]}$.

近年来, 随着深度学习技术的不断完善, 研究 人员结合深度学习技术对图像进行 SR 重建, 与传 统的 SR 技术相比, 在深度学习模式下能够恢复更 多的纹理细节. 但是, 目前深度学习下的 SR 技术 仍主要有以下 2 点问题: (1) 对 LR 图像的高频信息 提取问题; (2) 重建图像的整体表达是否真实.

针对 SR 技术的现有问题，本文提出一种基于 多感受野拉普拉斯生成对抗网络 (generative adversarial networks, GAN)的单幅图像 SR 算法, 并 取得了令人满意的实验结果. 本文主要通过以下 几个方面对算法结构进行设计:

(1) 提出可分离拉普拉斯滤波;

(2) 利用多感受野卷积;

(3) 提出复合残差密集块 (composite residual dense block, CRDB);

(4) 提出多维软标签 GAN.

\section{1 相关工作}

在最初的研究算法中, SR 使用线性、双三次插 值和 Lanczos 滤波算法 ${ }^{[6]}$ 进行单幅图像 SR 重建 (single image SR, SISR), 主要通过图像信息中包 含的视觉先验信息填充细节, 重建速度快, 但是对 内容的捕捉不够敏感，纹理细节不够理想. 在基于 学习的 SR 重建中, 随机森林算法 ${ }^{[7]}$ 取得了较好的
效果. 由于深度学习 ${ }^{[8]}$ 的进步, 图像 SR 开始尝试 深度学习的算法. 由于卷积神经网络的强大学习 能力, Dong 等 ${ }^{[9]}$ 提出了深度卷积网络下的 SR(image super-resolution using deep convolutional networks SRCNN)重建算法, 使用 3 个不同的卷积 层对图像进行 SR 重建, 开辟了神经网络 SR 重建 的道路. Kim 等 ${ }^{[10]}$ 受到 VGG 网络的影响, 在 SRCNN 的基础上加深了网络层次, 并使用了残差 学习, 提出了超深卷积网络 SR 重建算法(superresolution using very deep convolutional networks, VDSR). Tai 等 ${ }^{[11]}$ 采用多路残差和递归网络结构, 在增加网络层数的同时, 减少网络参数, 提出了深 度递归残差网络(deep recursive residual network, DRRN)对图像进行 SR 重建, 它比 SRCNN 和 VDSR 重建速度更快, 重建质量更优. 为了把握好 图像信息, Lai 等 ${ }^{[12]}$ 通过设计拉普拉斯金字塔网络 和一种新的损失函数, 提出的 LapSRN 能快速、准 确地对图像进行 SR 重建. Haris 等 ${ }^{[13]}$ 利用多个上下 采样模块增加 SR 的特征, 并通过反馈网络不同深 度的错误自纠正特征以构建输出, 提出了深度反向 投影网络(deep back-projection networks, DBPN)算 法用于 SR 重建. Li 等 ${ }^{[14]}$ 通过设计密集连接型反馈 块重用特征资源，并有效地处理反馈信息流，提出 了采用反馈机制的图像 SR 反馈网络(feedback network for image super-resolution, SRFBN).

$\mathrm{GAN}^{[15]}$ 为图像处理提供了新的研究方向. 尽 管 GAN 难以训练, 但是目前 GAN 与图像处理的结 合已被广泛应用, 如图像的风格转换 ${ }^{[16]}$ 、生成 ${ }^{[17]}$ 、 修复 ${ }^{[18]}$ 以及 SR 重建 ${ }^{[19]}$. Ledig 等 ${ }^{[20]}$ 提出了基于生 成对抗的单幅图像 SR 重建算法(super-resolution using a generative adversarial network, SRGAN) ${ }^{[20]}$, 在其模型中改进了 $\mathrm{He}$ 等 $^{[21]}$ 提出的残差结构, 并在 其中使用了批归一化(batch normalization, BN) ${ }^{[22]}$ 技术. BN 可以使训练更加容易, 最终取得了非常 好的效果, 但是其对于峰值信噪比 (peak signal-to-noise ratio, PSNR)和结构相似性没有较好的 量化结果. Wang 等 ${ }^{[23]}$ 改进了 SRGAN, 去除了残差 机制中的 $\mathrm{BN}$, 并将基础残差块改为密集残差中的 残差块(residual in residual dense block, RRDB) ${ }^{[23]}$, 提出了 ESRGAN ${ }^{[23]}$, 使重建效果较 SRGAN 有所 提高. Zhang 等 ${ }^{[24]}$ 提出的用于图像超分辨率的深度 展开网络(deep unfolding network for image super-resolution, USRNet)是基于深度学习和模型学 习的算法, 通过预训练多个降采样恢复模型, 用 
GAN 的算法进行训练, 对重建过程中特殊模糊的 处理有很好的效果, 对纹理的提取也取得了较大 的突破. 足够的、真实的纹理细节能提高图像的整 体质量，其可以通过纹理重建区分相似的 LR 图像. 在纹理细节补充方面, 可以通过损失函数对纹理 进行细化, 如 Ledig 等 ${ }^{[20]}$ 在 SRGAN 中提出的感知 损失，利用图像的高层特征作为损失依据，以提取 更多纹理; 还可通过特定的网络结构细化纹理, 如 在 $\mathrm{GAN}^{[15]}$ 中, 纹理通过其特有的对抗损失而变得 更加充盈; 也可通过一些特殊的网络层丰富纹理, 如 Wang 等 ${ }^{[25]}$ 在空间特征转换 GAN (spatial feature transform GAN, SFTGAN) ${ }^{[25]}$ 中提出了空间特征转 换 ${ }^{[25]}$ 层，通过先验类别丰富纹理信息.

上述算法主要有 3 个问题叹待解决: (1) 重建 图像的纹理细节问题, 算法需要从 LR 中提取更多 的细节; (2) 重建图像真实性的问题，重建的图像 不能只追求纹理细节，还要平衡纹理与整体图像 的搭配, 才能使得重建图像更加真实; (3) 使用 GAN 时网络难以训练. 基于以上 3 个问题，本文提 出了基于多感受野拉普拉斯 GAN 的 SISR 算法, 其 先通过拉普拉斯滤波对图像进行加强，再结合多 感受野卷积和 CRDB 提取和传递特征, 最后通过
软标签 GAN 优化 SR 重建结果. 本文重建结果拥 有丰富的纹理细节, 并且细节的处理和整体的搭 配也更加真实.

\section{2 网络模块结构}

\section{1 可分离拉普拉斯滤波和多感受野特征提取}

本文采用拉普拉斯算子对二维图像进行滤波, 其可以检测到图像中灰度突变的区域，将滤波后 的特征与原特征叠加, 可以使图像特征得到加强. 在滤波之前，利用 $1 \times 1$ 卷积对输人作非线性变换， 让网络一定程度上可以自主选择输人信息，丰富 输人的特征，使变换后的输人拥有更多的纹理信 息, 能更容易被卷积操作提取, 并配合残差连接增 加输人的多样性. 为了加速检测效果, 采用可分离 拉普拉斯滤波, 可分离式卷积 ${ }^{[26]}$ 将原始特征分离 后再卷积, 可提高运行速度. 为了能检测到更多的 特征, 本文对输人的图像进行了多感受野的特征 提取, 多感受野卷积可以对图像特征进行多角度 提取, 使重建图像的纹理细节更加丰富. 可分离拉 普拉斯滤波模块和多感受野特征提取分别如图 1 和图 2 所示.

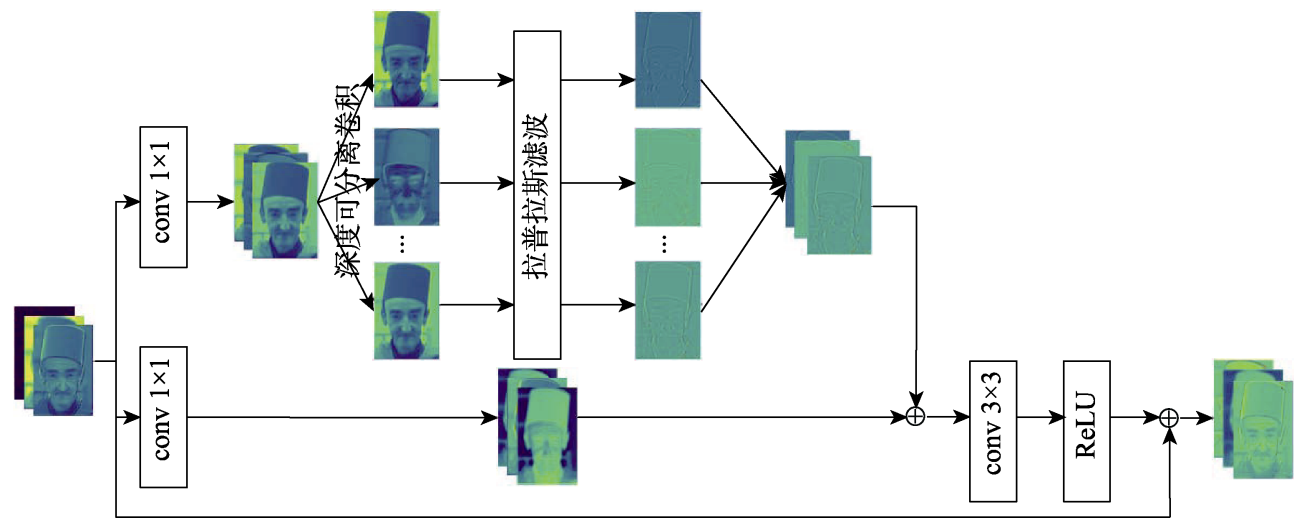

图 1 可分离拉普拉斯滤波模块

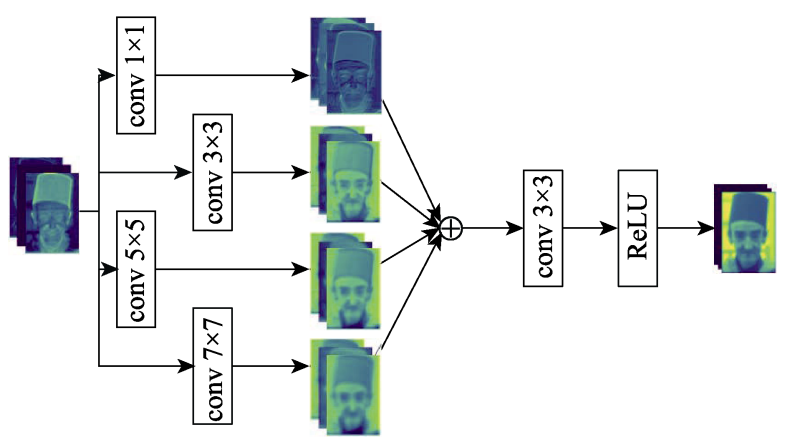

图 2 多感受野特征提取

\subsection{CRDB}

在深度网络中, 如果不采取特别措施, 初始特
征会随着层数的增加而逐渐消失, 为了使始特征 在每层能够有所保留, 本文采用了 CRDB 连接嵌 套了可分离拉普拉斯滤波和多感受野特征提取, 使各级特征在深度网络中的传递得到保障, 多层 CRDB 构成的网络可以拥有更加充足的图像特征. 其结构如图 3 所示.

\section{3 多维软标签 GAN}

$\mathrm{GAN}^{[15]}$ 从提出起就应用于图像处理中，一般 的 GAN 拥有一个生成器和一个判别器, 普通的判 别网络使用一维的硬标签计算损失, 难以训练且 训练效果不好. 本文中判别网络使用多维软标签 


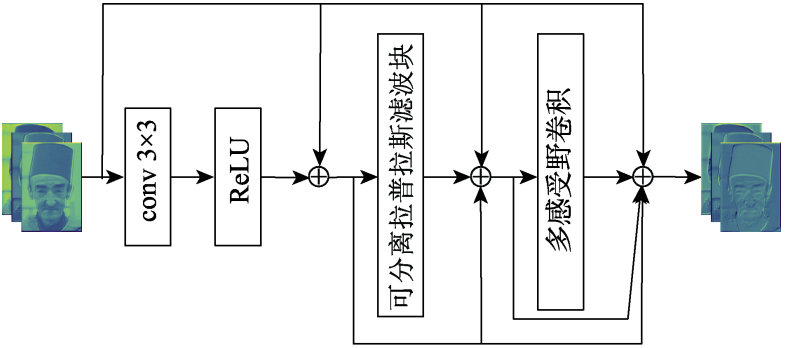

图 3 CRDB 结构

结构, 使判别器拥有一定的错误承受区间, 使其更 容易学习到 HR 和 SR 的差别, 因此也能够更好地 优化整个 GAN.

\section{3 本文算法}

图像 SR 的目标是将 $\mathrm{LR}$ 图像 $\boldsymbol{I}^{\mathrm{LR}}$ 经过 $\mathrm{SR}$ 过程 得到 $\mathrm{HR}$ 图像 $\boldsymbol{I}^{\mathrm{SR}}$. 本文提出基于多感受野拉普拉 斯 GAN 的 SISR 算法, 利用拉普拉斯滤波对纹理加 强, 利用多感受野多方位提取特征.二者经过滤波 重整后并人残差网络加强图像纹理信息, 以便获 得更多的图像信息. 另外, 由于归一化会影响图像 色彩的表达, 本文生成器中并未使用 BN. 为了使 模型的重建效果更好, 采用 CRDB 汇聚并加强各 个阶段的特征, 对每小层特征加权连接, 能够使最 初的特征也能参与最后的运算, 而又可以通过训 练更新参与运算的比例, 达到最佳的效果.

本文在生成器的末端使用上采样模块, 进而 使大量的运算都集中于低分辨率部分，从而大大 降低了运算量, 提升了计算速度. 本文将判别器的 图像质量评估结果映射为 10 维, 表示输人图像的 得分, 并使用软标签的方式, 希望生成图像的判定 得分区间为 $0 \sim 2$, 而真实图像的得分区间为 $7 \sim 9$, 这样可以使对抗网络更容易训练. 对于损失函数, 本文预训练采用 $L_{1}$ 损失和 VGG 低层特征作为损失 函数, 训练的主要损失函数由 VGG 最后一个卷积 层特征承担, VGG 网络的高层特征可以使重建的 图像更加精细, 再搭配 Charbonnier 损失对图像总 体进行约束，以提高重建效果的真实性.

本文利用 GAN 对图像进行超分辨率重建，给 定一个 $I^{\mathrm{LR}}$, 要求训练好的生成网络 $G$ 得到一个 $I^{\mathrm{SR}}$, 所以本文的目标之一就是训练得到一个生成 网络参数集

$$
\theta_{G}=\operatorname{argmin} \frac{1}{N} \sum_{i=1}^{N} \mathrm{IR}^{\mathrm{SR}}\left(G_{\theta_{G}}\left(\boldsymbol{I}_{i}^{\mathrm{LR}}\right), \boldsymbol{I}_{i}^{\mathrm{HR}}\right)
$$

其中, $l^{\mathrm{SR}}$ 表示生成 HR 图像与原 HR 图像之间的损
失; $G_{\theta_{G}}$ 表示当前最优参数生成网络参数集的生 成网络; $N$ 表示训练样本空间的大小; $I_{i}^{\mathrm{LR}}$ 表示第 $i$ 个 LR 训练样本, $i=1,2,3, \cdots, N ; \boldsymbol{I}_{i}^{\mathrm{HR}}$ 表示第 $i$ 个 $\mathrm{HR}$ 训练样本, $i=1,2,3, \cdots, N ;\left(\boldsymbol{I}_{i}^{\mathrm{LR}}, \boldsymbol{I}_{i}^{\mathrm{HR}}\right)$ 表示一个 配对训练集.

\subsection{GAN 结构}

$\mathrm{GAN}^{[15]}$ 拥有一个生成器和一个判别器, 对于 这 2 个独立的模块, 整个网络希望统一 2 个模块的 优化方向, 但是对抗的结构否定了统一优化目标 的存在, 普通 GAN 的优化目标为

$$
\begin{aligned}
& \min _{G} \max _{D} V(D, G)=\mathbb{E}_{\boldsymbol{I}^{\mathrm{HR}} \sim P_{\text {train }}\left(I^{\mathrm{HR}}\right)}\left[\log D\left(\boldsymbol{I}^{\mathrm{HR}}\right)\right]+ \\
& \mathbb{E}_{\boldsymbol{I}^{\mathrm{SR}} \sim P_{G\left(I^{\mathrm{LR}}\right)}}\left[1-\log D\left(G\left(\boldsymbol{I}^{\mathrm{LR}}\right)\right)\right]
\end{aligned}
$$

其中, $G$ 表示生成网络; $G\left(I^{\mathrm{LR}}\right)$ 表示 $\mathrm{LR}$ 输人生 成网络后得到的生成结果; $D$ 表示判别网络; $D\left(I^{\mathrm{HR}}\right)$ 用于判别原训练集中高分辨率图像是否 为真实图像; $D\left(G\left(I^{\mathrm{LR}}\right)\right)$ 用于判别生成器生成的 假图是否足够真实; $\mathbb{E}_{I^{\mathrm{HR}} \sim P_{\mathrm{train}}\left(I_{\mathrm{HR}}\right)}$ 表示输入图像属 于原训练集中 $\mathrm{HR}$ 图像的概率期望, $\mathbb{E}_{I^{\mathrm{SR}} \sim P_{G\left(I^{\mathrm{LR}}\right)}}$ 表 示输人图像属于生成器根据 LR 图像所生成图像的 概率期望. 本文对网络的优化目标进行了修改, 为 了清晰可见, 本文将优化目标拆为生成器和判别 器 2 个部分. 判别器的优化目标为

$$
\begin{aligned}
& \min _{D} V(D, G)= \\
& \mathbb{E}_{\boldsymbol{I}^{\mathrm{HR}} \sim P_{\text {train }}\left(I^{\mathrm{HR}}\right)}\left|\operatorname{rand}(a, b)-\arg \max \left(D\left(I^{\mathrm{HR}}\right)\right)\right|+ \\
& \mathbb{E}_{I^{\mathrm{SR}} \sim P_{G\left(I^{\mathrm{LR}}\right)}}\left|\operatorname{rand}(c, d)-\arg \max \left(D\left(G\left(I^{\mathrm{LR}}\right)\right)\right)\right|(3)
\end{aligned}
$$

其希望对生成图像给予相对较低的分, 对原 HR 图 像给予相对较高的分; 而生成器则期望每幅生成 的图像都能获得较高的分, 优化目标为

$$
\begin{aligned}
& \min _{G} V(D, G)= \\
& \mathbb{E}_{I^{\mathrm{SR}} \sim P_{G}\left(I^{\mathrm{LR}}\right)}\left|\operatorname{rand}(a, b)-\arg \max \left(D\left(G\left(I^{\mathrm{LR}}\right)\right)\right)\right|(4)
\end{aligned}
$$

其中, $a$ 和 $b$ 表示较高分所对应的区间; $c$ 和 $d$ 表示 较低分对应的区间.

为了达到上面的优化目标, 如图 4 所示, 在生 成器的设计中, 经过 $3 \times 3$ 的拉普拉斯滤波器提取 出轮廓信息, 再累加原特征, 以加强图像的纹理信 息. 之后通过提取多感受野特征, 得到更加完全的 特征和纹理信息. 最后使用 CRDB, 保证了特征在 深层网络的高质量传递. 对于每个拉普拉斯模块, 先让输人特征图通过一个 $1 \times 1$ 卷积层对原特征图 


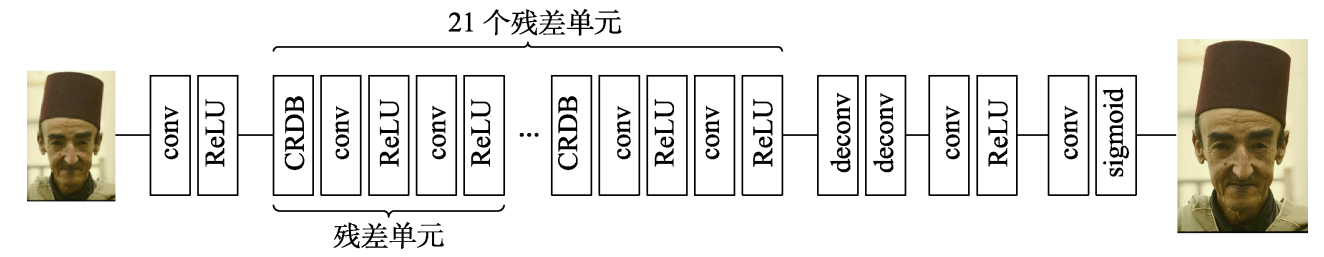

图 4 生成器结构

作非线性变换, 再通过一个 $3 \times 3$ 卷积输出到多感 受野特征提取模块中; 使用拉普拉斯滤波时, 本文 选择深度可分离卷积进行滤波, 深度可分离卷积 可以提高运算效率，降低时间复杂度. 对经过加强 后的特征图提取多感受野特征，将提取后的特征 相加, 使特征汇聚起来, 再通过密集连接输人下一 层网络. 在最后一个残差连接完成后, 生成网络将 接人 2 个连续的步长为 0.5 的上采样模块, 对图像 进行 4 倍上采样, 再通过卷积层, 将特征映射成一 幅图像, 得到生成网络的输出.
对于判别网络优化, 本文采用图像分类的原 则, 采用深度卷积网络 ${ }^{[27]}$, 对网络重建进行图像 10 分类, 每个分类代表一个打分等级, 判别器结构如 图 5 所示, 其中 $n 256 s 1$ 代表输出卷积步长为 1 , 通 道数为 256. 一个卷积模块中有 2 个卷积层, 每个卷 积层后使用 LeakyReLU 激活, 2 个卷积层之间加上 BN 层. 每 2 个卷积模块后会下采样一次, 特征图大 小减半, 通道数量增加为原来 2 倍. 特征提取完成 后, 将特征图扁平化输人 2 层全连接, 第 1 层采用 LeakyReLU 函数激活, 第 2 层直接映射为 10 维输出.

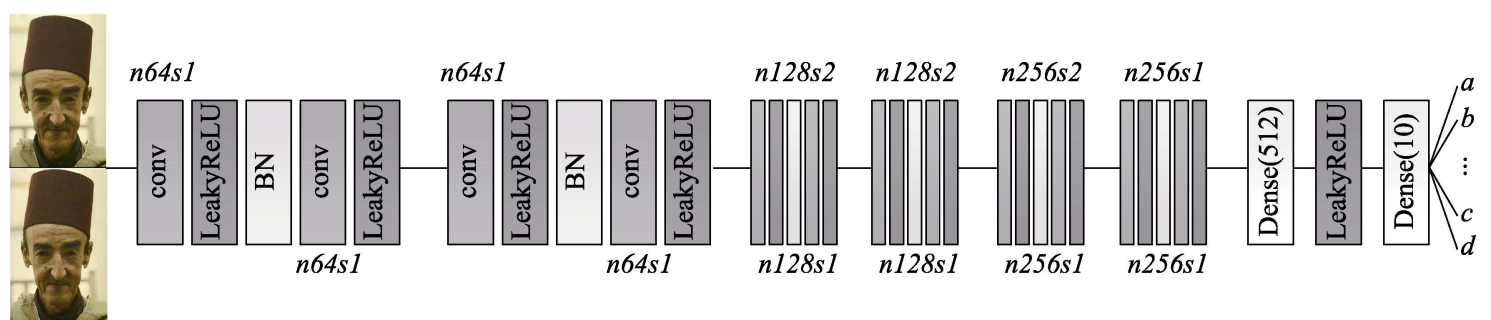

图 5 判别器结构

\section{2 感知损失函数}

感知损失函数定义的内容对算法的收敛、模型 的好坏影响重大，在传统模型中通常都是选择基 于像素级均方误差(mean square error, MSE) ${ }^{[28]}$ 作为 损失函数. Ledig 等 ${ }^{[20]}$ 改进了损失函数，采用 VGG 网络的高层次特征作为内容损失，再加权对抗损 失和正则化损失. 在此基础之上, 本文调整了损失 函数的搭配, 以提高感知质量.

\subsection{1 内容损失}

上文提到的像素级 MSE 损失函数的计算公式为

$$
l_{\mathrm{MSE}}^{\mathrm{SR}}=\frac{1}{W H} \sum_{x=1}^{W} \sum_{y=1}^{H}\left(G\left(\boldsymbol{I}^{\mathrm{LR}}\right)_{x, y}-\boldsymbol{I}_{x, y}^{\mathrm{HR}}\right)^{2}
$$

其中, $W$ 和 $H$ 分别表示图像的宽和高. MSE 在其拥 有较高 PSNR 的同时, 往往会使 SR 图像缺乏高频 信息, 纹理不够明显, 图像过于平滑.

在模型预训练时, 本文选择使用 $L_{1}$ 损失作为 预训练损失函数之一. $L_{1}$ 比 $L_{2}$ 损失能避免生成过 于平滑的图像，且能快速地得到图像特征提取的 效果. $L_{1}$ 损失的计算式为

$$
l_{L_{1}}^{\mathrm{SR}}=\frac{1}{W H} \sum_{x=1}^{W} \sum_{y=1}^{H}\left|G\left(\boldsymbol{I}^{\mathrm{LR}}\right)_{x, y}-\mathbf{I}_{x, y}^{\mathrm{HR}}\right|
$$

本文基于 Ledig 等 ${ }^{[20]}$ 和 Wang 等 ${ }^{[23]}$ 的思想, 选 择预训练好的 VGG19 网络用于高级特征提取, 选 择使用其高层激活前的特征作为计算感知损失的 依据. 用 $\varphi(x)$ 表示图像 $x$ 输人 VGG19 网络后得到 的特征图, 本文选择的内容损失函数计算式为

$$
\begin{aligned}
& l_{\mathrm{VGG}_{i, j}}^{\mathrm{SR}}=\frac{1}{W_{\mathrm{VGG}_{i, j}} H_{\mathrm{VGG}_{i, j}}} \times
\end{aligned}
$$

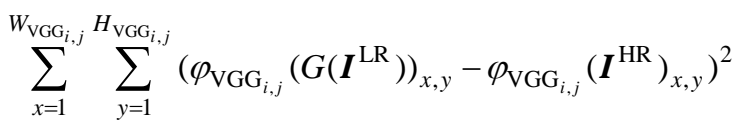

其中, $W_{\mathrm{VGG}_{i, j}}$ 和 $H_{\mathrm{VGG}_{i, j}}$ 表示 VGG19 网络第 $i$ 层第 $j$ 个卷积输出特征图的宽和高. 式(7)用于计算生成 图像与原 HR 图像经过特征提取后的欧几里得度 量. 在损失函数的使用上, 本文在预训练时选择 $\mathrm{VGG}_{2,2}$ 作为损失函数的组成部分, 在模型训练时 选择 $\mathrm{VGG}_{5,4}$ 作为损失函数的主要部分. 
实际上，在模型的训练过程中，本文还使用 Charbonnier 损失函数规避图像生成过程中产生的 异常点, 使生成图像相对更加真实. Charbonnier 损 失 $l_{\mathrm{ch}}^{\mathrm{SR}}$ 计算公式为

$$
\begin{aligned}
& \boldsymbol{\Delta}=\boldsymbol{I}^{\mathrm{HR}}-\boldsymbol{I}^{\mathrm{SR}} \\
& l_{\mathrm{ch}}^{\mathrm{SR}}=\frac{1}{W H} \sum_{x=1}^{W} \sum_{y=1}^{H} \sqrt{\Delta_{x, y}^{2}+\epsilon^{2}}
\end{aligned}
$$

其中, $W$ 和 $H$ 表示生成图像的宽和高; $\Delta$ 表示 $H R$ 图像与生成图像的差值矩阵; $A_{x, y}$ 表示在某个像 素点的差值; $\epsilon$ 表示超参数.

\subsection{2 生成损失}

除了内容损失以外, 本文还加人了 GAN 特有 的生成损失和对抗损失, 生成损失也能帮助模型 调优. 生成损失的定义是基于判别器的, 本文希望 生成器生成的图像能够欺骗判别器. 生成损失计 算公式为

$$
\begin{aligned}
& I_{\mathrm{Gen}}^{\mathrm{SR}}= \\
& \frac{1}{N} \sum_{n=1}^{N}\left|\operatorname{rand}(a, b)-\arg \max \left(D\left(G\left(I^{\mathrm{LR}}\right)\right)\right)\right|
\end{aligned}
$$

其中, $D\left(G\left(I^{\mathrm{LR}}\right)\right)$ 表示生成分为 $\mathrm{HR}$ 图像的类别, 生成器希望被分为 $\mathrm{HR}$ 图像类别; $N$ 表示生成图像 的样本量. 为了得到更好的 SR 结果, 本文选择最 小化生成损失.

\subsection{3 对抗损失}

GAN 与普通的神经网络差别为多了一个对抗 网络, 本文的判别器也需要优化. 判别器优化是一 个独立的工作, 有了好的判别器才能提供有效的 生成损失, 生成器才会进步, 判别器的优化至关重 要. 判别器的损失函数为

$$
\begin{aligned}
& \varsigma_{n}=\left|\operatorname{rand}(a, b)-\arg \max \left(D\left(I^{\mathrm{HR}}\right)\right)\right|+ \\
& \left|\operatorname{rand}(c, d)-\arg \max \left(D\left(G\left(I^{\mathrm{LR}}\right)\right)\right)\right| \\
& l_{\mathrm{D}}^{\mathrm{SR}}=\frac{1}{N} \sum_{n=1}^{N} \varsigma_{n}
\end{aligned}
$$

其中, $N$ 表示样本集大小; $a, b$ 和 $c, d$ 分别表示判 别器判为高质量和低质量的软标签上下限.

\subsection{4 预训练与训练损失}

通过上面的损失介绍，本文预训练和训练损 失可依据上述缺失确定, 预训练只针对生成器训 练, 损失函数定义为

$$
l_{\mathrm{p}}^{\mathrm{SR}}=\alpha_{1} \mathrm{l}_{L_{1}}^{\mathrm{SR}}+\alpha_{2} \mathrm{~V}_{\mathrm{VGG}_{2,2}}^{\mathrm{SR}}
$$

训练包含生成器和判别器 2 个模块, 故有 2 个损失, 判别器损失定义如式(12)所示, 训练时生成器损失 的定义为

$$
l^{\mathrm{SR}}=\gamma_{1} \mathrm{VGG}_{5,4}^{\mathrm{SR}}+\gamma_{2} l_{\mathrm{ch}}^{\mathrm{SR}}+\gamma_{3} l_{\mathrm{Gen}}^{\mathrm{SR}}
$$

其中, $\alpha, \gamma$ 表示各损失分量的权重系数. 预训练选 择低层次特征 $\mathrm{VGG}_{2,2}$ 作为损失函数的一部分可以 为重建图像提供更多的重建细节, 但是不够精细; 在正式训练过程中, 采用高层次特征 $\mathrm{VGG}_{5,4}$ 作为 损失函数可以获得更为精细的图像特征, 让图形 看起来更真实.

\section{3 整体架构及性能分析}

本文算法中可分离拉普拉斯滤波单元用于纹 理凸显, 其计算速度快, 优化效率高; 多感受野卷 积可以准确把握图像纹理，并全方位提取特征; CRDB 解决了深层网络特征传递问题; GAN 的训 练上选用多维软标签, 在保持 GAN 训练效果的同 时降低了训练的复杂性. 本文整个生成网络参数 约有 $10.2 \times 10^{6}$, 参数数量远小于 ESRGAN 的 $16.7 \times 10^{6}$ 和 USRNet 的 $17.0 \times 10^{6}$; 本文算法运行速 度更快, 执行效率更高. 在重建结果上, 与 ESRGAN 等传统 GAN 算法相比, 本文在拥有更加 丰富的纹理细节的同时, 具有更加真实的重建效 果; 与最新的 USRNet 相比, USRNet 过分追求细节 纹理的重建，而不考虑细节与图像的整体搭配，显 得图像不够真实, 而本文恰好解决了细节与整体 的问题，在拥有足够纹理的同时使图像更加自然.

\section{4 实验及结果分析}

\section{1 实验环境}

本文算法实验的硬件环境为 Intel Xeon CPU E5-2620 v4 x32, 64GB 内存, TITAN XP GPU; 使用 Ubuntu 16.04 LTS 操作系统, 以 TensorFlow 作为深 度学习框架.

\section{2 实验数据与量化标准}

本文选择常用的 SR 数据集 Set $5^{[29]}$, Set $14^{[30]}$, BSD $100^{[31]}$ 和 General $100^{[32]}$ 进行实验. 为了比较的 公平性, 本文采用 PI (perceptual index) ${ }^{[33]}$ 衡量算法 的优劣, PSNR 作为质量评价参考指标. PI 通过无 参考评价指标 $\mathrm{Ma}$ 's score $(\mathrm{Ma})^{[34]}$ 和 $\mathrm{NIQE}^{[35]}$ 对图像 的感知质量进行度量, 即

$$
\mathrm{PI}=\frac{(10-\mathrm{Ma})+\mathrm{NIQE}}{2} .
$$

较低的感知指数代表较好的感知质量, 具有 较全面的细节. PSNR 通过计算最大像素值平方与 图像均方误差商的对数来评估图像的质量, 其数 值越高, 代表图像在像素距离上越接近; 数值较 低, 代表图像存在失真. 参与比较的算法有文献 
[9,12,20,23-24,36]等，PSNR 使用 scikit-image 中的 measure 包进行计算.

\section{3 训练细节及参数设置}

本文的训练使用的数据集为 $\mathrm{Div} 2 \mathrm{~K}^{[37]}$ 和 Flickr2K ${ }^{[38]}$. 首先将每幅高清大图随机裁剪成 20 个196×196 像素的小图作为 HR 数据, 再将其通过 BICUBIC 算法下采样为对应 LR 图像，下采样因子 为 4. 对于 $\mathrm{HR}$ 重建的过程, 本文下采样使用的卷 积核大小均为 $3 \times 3$, 上采样步长为 0.5 , 生成器使 用 21 个相同的 CRDB, 判别器模型如图 5 所示. 训 练使用 $\operatorname{Adam}^{[39]}$ 优化器进行优化, 其中 $\beta_{1}=0.9$. 预训练一共训练 100 轮, 设置初始学习率为 0.0001 , 学习率每 20 轮变为原来的一半. 正式训 练对生成器和判别器使用不同的学习率进行更新, 设置生成器的学习率为 0.0001 , 判别器的学习率
为 0.0004 . 生成器和判别器交替训练, 训练次数 100 轮, 每 20 轮学习率减少为原来的一半. 训练时 每个 batch 有 16 组数据同时进行计算, Charbonnier 损失函数中 $\epsilon=0.001$. 设置预训练损失函数中 $\alpha_{1}=0.9, \alpha_{2}=0.1$, 生成器损失函数中 $\gamma_{1}=1$, $\gamma_{2}=0.01, \gamma_{3}=0.001$. 本文的超参数设置都是通 过前人的经验对参数大小进行方向上的估计, 根 据每次实验结果对超参数进行反复调整，当设置 如上所示的超参数时, 预训练能产生更多的细节, 训练也能获得更加精细的重建图像.

\section{4 量化结果}

模型训练完成后, 本文选择与各经典、流行算 法以及最新算法进行比较, 在 Set5, Set14, BSD100 和 General 100 数据集上生成图像的量化比较如表 1 所示.

表 18 种算法在各数据集上的量化比较

\begin{tabular}{|c|c|c|c|c|c|c|c|c|}
\hline \multirow{2}{*}{ 算法 } & \multicolumn{2}{|c|}{ Set5 } & \multicolumn{2}{|c|}{ Set14 } & \multicolumn{2}{|c|}{ BSD100 } & \multicolumn{2}{|c|}{ General100 } \\
\hline & PSNR/dB & PI & $\mathrm{PSNR} / \mathrm{dB}$ & PI & $\mathrm{PSNR} / \mathrm{dB}$ & PI & $\mathrm{PSNR} / \mathrm{dB}$ & PI \\
\hline BICUBIC & 25.10 & 6.58 & 26.25 & 7.03 & 30.03 & 7.60 & 25.15 & 6.80 \\
\hline 文献[36] & $\underline{27.56}$ & 5.77 & 28.15 & 6.42 & 29.78 & 6.46 & 25.49 & 5.24 \\
\hline 文献[9] & 27.22 & 5.61 & $\underline{28.98}$ & 6.40 & 32.65 & 6.31 & $\underline{25.50}$ & 5.82 \\
\hline 文献[12] & 27.17 & 5.75 & 28.16 & 5.60 & 31.81 & 6.51 & 25.54 & 4.96 \\
\hline 文献[20] & 27.06 & 3.93 & 27.93 & 4.28 & 32.19 & 4.87 & 24.56 & 3.37 \\
\hline 文献[23] & 28.13 & 3.45 & 30.35 & 3.88 & $\underline{32.25}$ & 3.15 & 22.59 & 2.48 \\
\hline 文献[24] & 18.85 & $\underline{2.99}$ & 20.81 & $\underline{3.75}$ & 26.60 & 2.58 & 18.68 & 2.27 \\
\hline 本文 & 26.10 & 2.96 & 28.27 & 3.72 & 31.16 & $\underline{2.98}$ & 24.08 & 1.94 \\
\hline
\end{tabular}

注. 加粗表示最优指标, 下划线表示次优指标.

从表 1 中可以观察到, 本文算法在拥有最好 PI 量化结果的同时, PSNR 量化结果处于一个正常 范围之内; 而 USRNet 虽然拥有较好的 PI 结果, 但 是因为纹理过度提取, 图像失真, 导致 PSNR 结果 较低. 本文算法拥有丰富的纹理细节，重建合理， 质量更优. 图 6 图 9 所示为各算法在不同数据集
上细节的直观比较.

通过各算法的直观比较，容易发现 BICUBIC 算法的重建效果最差, 图像非常模糊. 文献[36]重 建效果较 BICUBIC 略好, 但结构不够清晰. 文献 [9]和文献[12]的重建图像纹理较 BICUBIC 和文献 [36]更为清晰, 但效果依旧不够好. 文献[20]的重

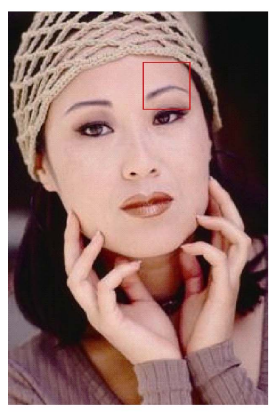

Set5_woman

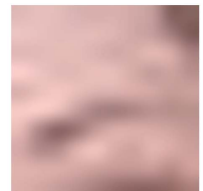

LR

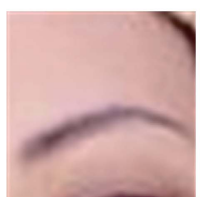

HR

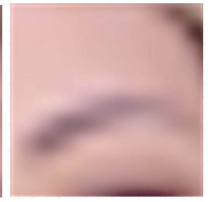

BICUBIC

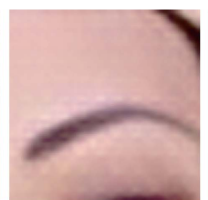

文献[20]

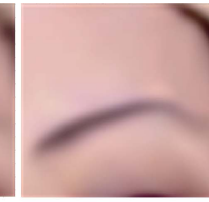

文献[36]

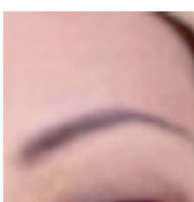

文献[23]

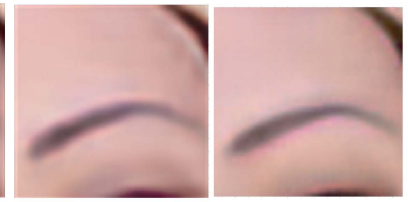

文献[9]

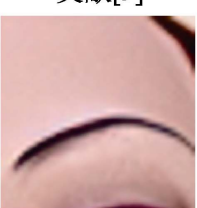

文献[24]

文献[12]
图 68 种算法在 Set5 数据集上的对比 


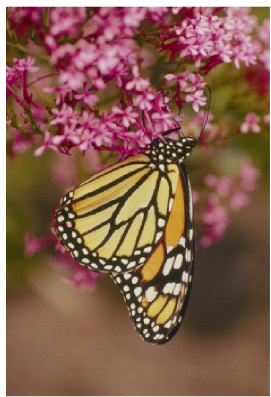

Set14 monarch

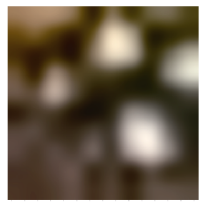

LR

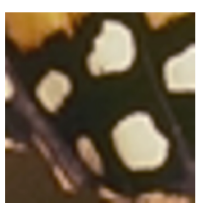

HR

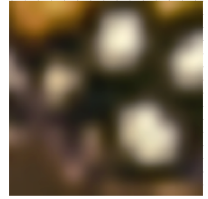

BICUBIC

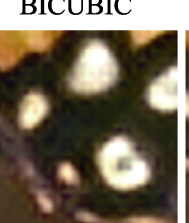

文献[20]

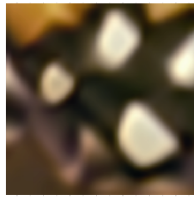

文献[36]

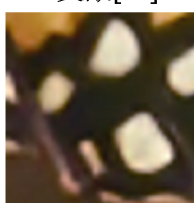

文献[23]

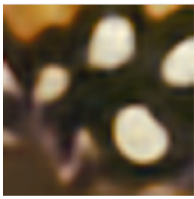

文献[9]

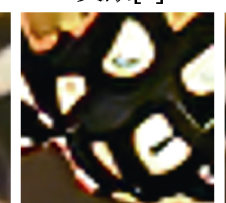

文献[24]

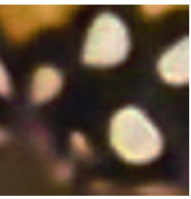

文献[12]

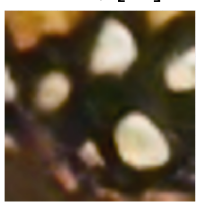

本文

图 78 种算法在 Set14 数据集上的对比

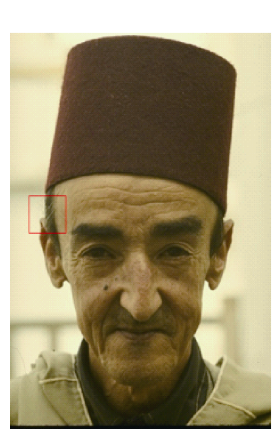

BSD100_189080

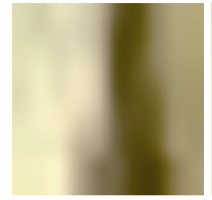

LR

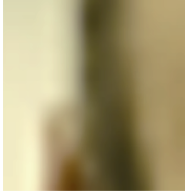

BICUBIC

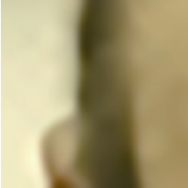

文献[36]

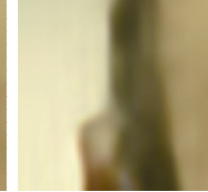

文献[9]

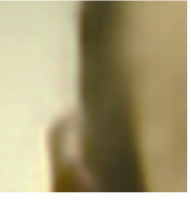

文献[12]
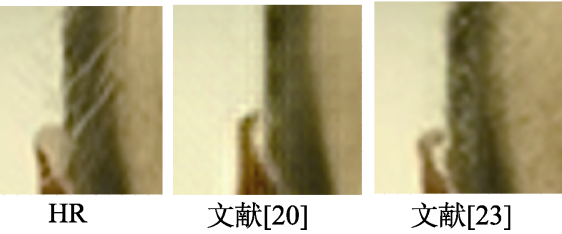

文献[23]

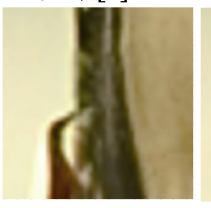

文献[24]

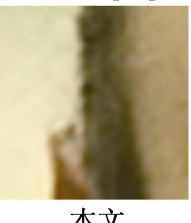

本文

图 88 种算法在 BSD100 数据集上的对比

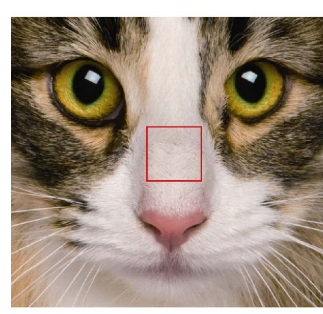

General100_im_016

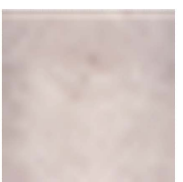

LR

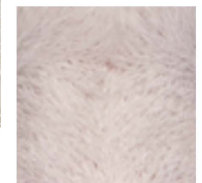

HR

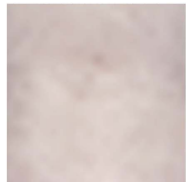

BICUBIC

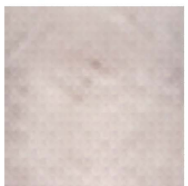

文献[20]

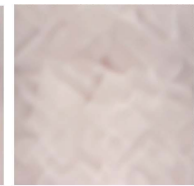

文献[36]

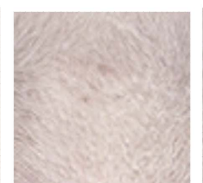

文献[23]

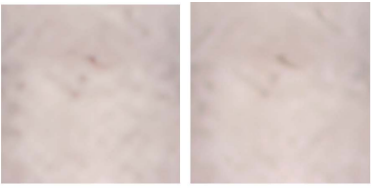

文献[9] 文献[12]

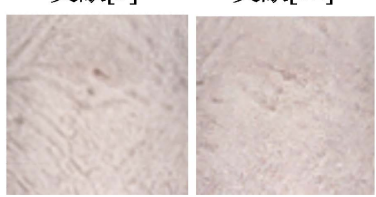

文献[24] 本文

图 98 种算法在 General100 数据集上的对比

建图像具有更多的纹理细节, 较前几种算法具有 更好的效果, 但是与文献[23]和文献[20]相比更加 模糊. 文献[24]色彩更加鲜艳, 但看起来不够真实, 并且产生了明显的伪细节. 比较来看, 本文算法在 纹理细节上具有优势, 在图像效果上也足够清晰, 细节与整体的搭配更加合理，比其他算法的重建 结果更加真实.

\section{5 实验小结}

通过实验对比，本文通过拉普拉斯滤波对图 像纹理进行加强, 使重建图像能够拥有足够的纹 理被提取; 通过多感受野特征提取和残差密集连 接的特征传递，使上采样阶段拥有丰富的纹理进
行重建; 使用多维软标签优化判别器, 使 GAN 能 够重建出更加真实的图像.

\section{5 结 语}

针对 SR 重建细节提取不够全面、重建图像不 够真实以及 GAN 难以训练等问题, 本文设计了一 个基于多感受野拉普拉斯的 GAN 进行 SR 重建, 它可以将 LR 图像重建为 HR, 使 SR 图像与原始的 HR 图像差别较小, 并且网络规模小, 运行速度快, 具有更优的结果. 通过数据的比较和直观的图像 展示，本文算法同时解决了纹理细节和整体搭配 
的问题，重建的图像显得更加清晰和真实.

由于拉普拉斯滤波模型的限制和网络层数的 发展，本文算法在某些纹理极其复杂的图像上重 建效果有一定的提升空间. 另外，随着深度学习领 域的发展，深度网络的运行速度也有极大的提高 潜力. 因此, 未来对 SR 的研究可以更加注重纹理 的提取, 并设计新的网络框架来提高重建效率。

\section{参考文献(References):}

[1] Chen Boyang, Gu Songyan. Research on super-resolution reconstruction of meteorological satellite remote sensing images[C] //Proceedings of the 28th Annual Meeting of the Chinese Meteorological Society. Beijing: Chinese Meteorological Society, 2011: 1-9(in Chinese)

(陈博洋, 谷松岩. 气象卫星遥感图像的超分辨率重建研究 [C] //第 28 届中国气象学会年会论文集. 北京: 中国气象学 会, 2011: 1-9)

[2] Shi W Z, Caballero J, Ledig C, et al. Cardiac image super-resolution with global correspondence using multi-atlas PatchMatch[C] //Proceedings of International Conference on Medical Image Computing and Computer-Assisted Intervention. Heidelberg: Springer, 2013: 9-16

[3] Song Dingyu. The research of video face recognition using super-resolution reconstruction based on multi-level FFD registration[J]. Laser Magazine, 2014, 35(12): 30-35(in Chinese) (宋定宇. 多级 FFD 融合超分辨率重建的视频人脸识别 [J]. 激光杂志, 2014, 35(12): 30-35)

[4] Harris J L. Diffraction and resolving power[J]. Journal of the Optical Society of America, 1964, 54(7): 931-936

[5] Freeman W T, Jones T R, Pasztor E C. Example-based super-resolution[J]. IEEE Computer graphics and Applications, 2002, 22(2): 56-65

[6] Duchon C E. Lanczos filtering in one and two dimensions[J]. Journal of Applied Meteorology and Climatology, 1979, 18(8): 1016-1022

[7] Wu Chengdong, Lu Ziwei, Yu Xiaosheng. Image super resolution reconstruction algorithm based on weighted random forest [J]. Control and Decision, 2019, 34(10): 2243-2248(in Chinese) (吴成东, 卢紫微, 于晓升. 基于加权随机森林的图像超分 辨率算法研究 [J]. 控制与决策, 2019, 34(10): 2243-2248)

[8] Ha V K, Ren J C, Xu X Y, et al. Deep learning based single image super-resolution: a survey[C] //Proceedings of International Conference on Brain Inspired Cognitive Systems. Heidelberg: Springer, 2018: 106-119

[9] Dong C, Loy C C, He K M, et al. Image super-resolution using deep convolutional networks[J]. IEEE Transactions on Pattern Analysis and Machine Intelligence, 2016, 38(2): 295-307

[10] Kim J, Lee J K, Lee K M. Accurate image super-resolution using very deep convolutional networks[C] //Proceedings of the IEEE Conference on Computer Vision and Pattern Recognition. Los Alamitos: IEEE Computer Society Press, 2016: 1646-1654

[11] Tai Y, Yang J, Liu X M. Image super-resolution via deep recursive residual network[C] //Proceedings of the IEEE Conference on Computer Vision and Pattern Recognition. Los Alamitos: IEEE Computer Society Press, 2017: 3147-3155

[12] Lai W S, Huang J B, Ahuja N, et al. Deep Laplacian pyramid networks for fast and accurate super-resolution[C] //Proceedings of the IEEE Conference on Computer Vision and Pattern Recognition. Los Alamitos: IEEE Computer Society Press, 2017: 624-632

[13] Haris M, Shakhnarovich G, Ukita N. Deep back-projection networks for super-resolution[C] //Proceedings of the IEEE Conference on Computer Vision and Pattern Recognition. Los Alamitos: IEEE Computer Society Press, 2018: 1664-1673

[14] Li Z, Yang J L, Liu Z, et al. Feedback network for image super-resolution[C] //Proceedings of the IEEE/CVF Conference on Computer Vision and Pattern Recognition. Los Alamitos: IEEE Computer Society Press, 2019: 3867-3876

[15] Goodfellow I J, Pouget-Abadie J, Mirza M, et al. Generative adversarial networks[OL]. [2020-11-05]. https://arxiv.org/abs/ 1406.2661

[16] Zhu J Y, Park T, Isola P, et al. Unpaired image-to-image translation using cycle-consistent adversarial networks[C] //Proceedings of the IEEE International Conference on Computer Vision. Los Alamitos: IEEE Computer Society Press, 2017: 2223-2232

[17] Xu T, Zhang P C, Huang Q Y, et al. AttnGAN: fine-grained text to image generation with attentional generative adversarial networks[C] //Proceedings of the IEEE Conference on Computer Vision and Pattern Recognition. Los Alamitos: IEEE Computer Society Press, 2018: 1316-1324

[18] Chen Junzhou, Wang Juan, Gong Xun. Face image inpainting using cascaded generative adversarial networks[J]. Journal of University of Electronic Science and Technology of China, 2019, 48(6): 910-917(in Chinese)

(陈俊周, 王娟, 龚勋. 基于级联生成对抗网络的人脸图像 修复 $[J]$. 电子科技大学学报, 2019, 48(6): 910-917)

[19] Yang J C, Wright J, Huang T S, et al. Image super-resolution via sparse representation[J]. IEEE Transactions on Image Processing, 2010, 19(11): 2861-2873

[20] Ledig C, Theis L, Huszár F, et al. Photo-realistic single image super-resolution using a generative adversarial network[C] //Proceedings of the IEEE Conference on Computer Vision and Pattern Recognition. Los Alamitos: IEEE Computer Society Press, 2017: 4681-4690

[21] He K M, Zhang X Y, Ren S Q, et al. Deep residual learning for image recognition[C] //Proceedings of the IEEE Conference on Computer Vision and Pattern Recognition. Los Alamitos: IEEE Computer Society Press, 2016: 770-778

[22] Ioffe S, Szegedy C. Batch normalization: accelerating deep network training by reducing internal covariate shift $[\mathrm{C}]$ //Proceedings of the 32nd International Conference on Machine Learning. Lille: PMLR, 2015: 448-456

[23] Wang X T, Yu K, Wu S X, et al. ESRGAN: enhanced super-resolution generative adversarial networks[C] //Proceedings of European Conference on Computer Vision. Heidelberg: Springer, 2018: 63-79

[24] Zhang K, van Gool L, Timofte R. Deep unfolding network for image super-resolution[C] //Proceedings of the IEEE/CVF 
Conference on Computer Vision and Pattern Recognition. Los Alamitos: IEEE Computer Society Press, 2020: 3217-3226

[25] Wang X T, Yu K, Dong C, et al. Recovering realistic texture in image super-resolution by deep spatial feature transform[C] //Proceedings of the IEEE Conference on Computer Vision and Pattern Recognition. Los Alamitos: IEEE Computer Society Press, 2018: 606-615

[26] Howard A G, Zhu M L, Chen B, et al. MobileNets: efficient convolutional neural networks for mobile vision applications[OL]. [2020-11-05]. https://arxiv.org/abs/1704.04861

[27] Al-Saffar A A M, Tao H, Talab M A. Review of deep convolution neural network in image classification[C] //Proceedings of the International Conference on Radar, Antenna, Microwave, Electronics, and Telecommunications. Los Alamitos: IEEE Computer Society Press, 2017: 26-31

[28] Shi W Z, Caballero J, Huszár F, et al. Real-time single image and video super-resolution using an efficient sub-pixel convolutional neural network[C] //Proceedings of the IEEE Conference on Computer Vision and Pattern Recognition. Los Alamitos: IEEE Computer Society Press, 2016: 1874-1883

[29] Bevilacqua M, Roumy A, Guillemot C, et al. Low-complexity single-image super-resolution based on nonnegative neighbor embedding[C] //Proceedings of the British Machine Vision Conference. Guildford: BMVA Press, 2012: 135-135

[30] Zeyde R, Elad M, Protter M. On single image scale-up using sparse-representations[C] //Proceedings of International Conference on Curves and Surfaces. Heidelberg: Springer, 2010: 711-730

[31] Martin D, Fowlkes C, Tal D, et al. A database of human segmented natural images and its application to evaluating segmentation algorithms and measuring ecological statistics $[\mathrm{C}]$ //Proceedings of the 8th IEEE International Conference on
Computer Vision. Los Alamitos: IEEE Computer Society Press, 2001: 416-423

[32] Dong C, Loy C C, Tang X. Accelerating the super-resolution convolutional neural network[C] //Proceedings of European Conference on Computer Vision. Heidelberg: Springer, 2016: 391-407

[33] Blau Y, Mechrez R, Timofte R, et al. The 2018 PIRM Challenge on Perceptual Image Super-Resolution[C] //Proceedings of European Conference on Computer Vision. Heidelberg: Springer, 2018: 334-355

[34] Ma C, Yang C Y, Yang X K, et al. Learning a no-reference quality metric for single-image super-resolution[J]. Computer Vision and Image Understanding, 2017, 158: 1-16

[35] Mittal A, Soundararajan R, Bovik A C. Making a "completely blind” image quality analyzer[J]. IEEE Signal Processing Letters, 2013, 20(3): 209-212

[36] Huang J B, Singh A, Ahuja N. Single image super-resolution from transformed self-exemplars[C] //Proceedings of the IEEE Conference on Computer Vision and Pattern Recognition. Los Alamitos: IEEE Computer Society Press, 2015: 5197-5206

[37] Agustsson E, Timofte R. NTIRE 2017 challenge on single image super-resolution: dataset and study[C] //Proceedings of the IEEE Conference on Computer Vision and Pattern Recognition Workshops. Los Alamitos: IEEE Computer Society Press, 2017: 126-135

[38] Timofte R, Agustsson E, van Gool L, et al. NTIRE 2017 challenge on single image super-resolution: methods and results[C] //Proceedings of the IEEE Conference on Computer Vision and Pattern Recognition Workshops. Los Alamitos: IEEE Computer Society Press, 2017: 114-125

[39] Kingma D P, Ba J. Adam: a method for stochastic optimization[OL]. [2020-11-05]. https://arxiv.org/abs/1412.6980v4 